CDF/PUB/JET/PUBLIC/8548

\title{
STUDYING THE UNDERLYING EVENT AT CDF
}

\author{
R. D. FIELD*1 \\ Department of Physics, University of Florida, Gainesville, Florida, 32611, USA \\ *E-mail: rfield@phys.ufl.edu
}

\begin{abstract}
A study of "underlying event" in Run 2 at CDF is presented. Several PYTHIA 6.2 tunes (with multiple parton interactions) are examined and compared with HERWIG (without multiple parton interactions) and with the ATLAS PYTHIA tune (with multiple parton interactions) and they are extrapolated to the LHC.
\end{abstract}

Keywords: QCD, Jets, Hadron Collider.

In order to find "new" physics at a hadronhadron collider it is essential to have MonteCarlo models that simulate accurately the “ordinary" QCD events. To do this one must not only have a good model of the hard scattering part of the process, but also of "underlying event". The "hard scattering" component of the event consists of particles that result from the hadronization of the two outgoing partons (i.e. the initial two "jets") plus the particles that arise from initial and final state radiation (i.e. multijets). The "underlying event" consists of particles that arise from the "beam-beam remnants" and from multiple parton interactions (MPI). Of course, in a given event it is not possible to uniquely determine the origin of the outgoing particles and whatever observable one chooses to study inevitably receives contributions from both the hard component and the underlying event. In studying observables that are sensitive to the underlying event one learns not only about the "beam-beam remnants" and multiple parton interactions, but also about hadronization and initial and final state radiation.

The underlying event is an unavoidable background to most collider observables and a good understanding of it will lead to more precise measurements at the Tevatron and the LHC. For example, at the Tevatron both the inclusive jet cross section and the b-jet cross section depend sensitively on the underlying event. At CDF we are working to understand and model the underlying event at the Tevatron. We are also trying to extrapolate what we are learning at the Tevatron to the LHC. We use the topological structure of hadron-hadron collisions to study the underlying event. The direction of the leading calorimeter jet is used to isolate regions of $\eta-\phi$ space that are sensitive to the "underlying event". The direction of the leading jet, jet\#1, is used to define correlations in the azimuthal angle, $\Delta \phi$. The angle $\Delta \phi=\phi-\phi_{\text {jet\#1 }}$ is the relative azimuthal angle between a charged particle and the direction of jet\#1. The two "transverse" regions $60^{\circ}<\Delta \phi<120^{\circ}$ and $60^{\circ}<$ $-\Delta \phi<120^{\circ}$ are almost perpendicular to the plane of the hard 2-to-2 scattering and are therefore very sensitive to the underlying event. Furthermore, we consider two classes of events. We refer to events in which there are no restrictions placed on the second and third highest $\mathrm{P}_{\mathrm{T}}$ jets (jet\#2 and jet\#3) as "leading jet" events. Events with at least two jets with $\mathrm{P}_{\mathrm{T}}>15 \mathrm{GeV}$ where the leading two jets are nearly "back-to-back" $\left(\left|\Delta \phi_{12}\right|>150^{\circ}\right)$ with $\mathrm{P}_{\mathrm{T}}\left(\right.$ jet\#2)/ $\mathrm{P}_{\mathrm{T}}($ jet\#1 $)>0.8$ and $\mathrm{P}_{\mathrm{T}}($ jet\#3) $<$ $15 \mathrm{GeV}$ are referred to as "back-to-back" events. "Back-to-back" events are a subset of the "leading jet" events. The idea here is to suppress hard initial and final-state radiation thus increasing the sensitivity of the

\footnotetext{
${ }^{1}$ To appear in the proceedings of the XXXIII International Conference on High Energy Physics (ICHEP06).
} 
"transverse" region to the "beam-beam remnant" and the multiple parton scattering component of the "underlying event".

In addition, we define a variety of MAX and MIN "transverse" regions which helps separate the "hard component" from the underlying event. MAX (MIN) refer to the "transverse" region containing the largest (smallest) number of charged particles or the region containing the largest (smallest) scalar $\mathrm{p}_{\mathrm{T}}$ sum of particles. Since we will be studying regions in $\eta-\phi$ space with different areas, we construct densities by dividing by the area. For example, the charged particle density, $\mathrm{dN} / \mathrm{d} \eta \mathrm{d} \phi$, corresponds number of charged particle with $\mathrm{p}_{\mathrm{T}}>0.5 \mathrm{GeV} / \mathrm{c}$ per unit $\eta-\phi$, and

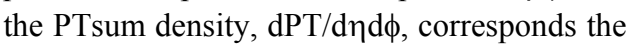
amount of charged particle $\left(\mathrm{p}_{\mathrm{T}}>0.5 \mathrm{GeV} / \mathrm{c}\right)$ scalar $\mathrm{p}_{\mathrm{T}}$ sum per unit $\eta-\phi$. The overall "transverse" region is the average of the "transMAX" and the "transMIN" region. One expects that "transMAX" will pick up the hardest initial or final-state radiation while both "transMAX" and "transMIN" should receive contributions from the underlying event. Hence, one expects "transMIN" to be
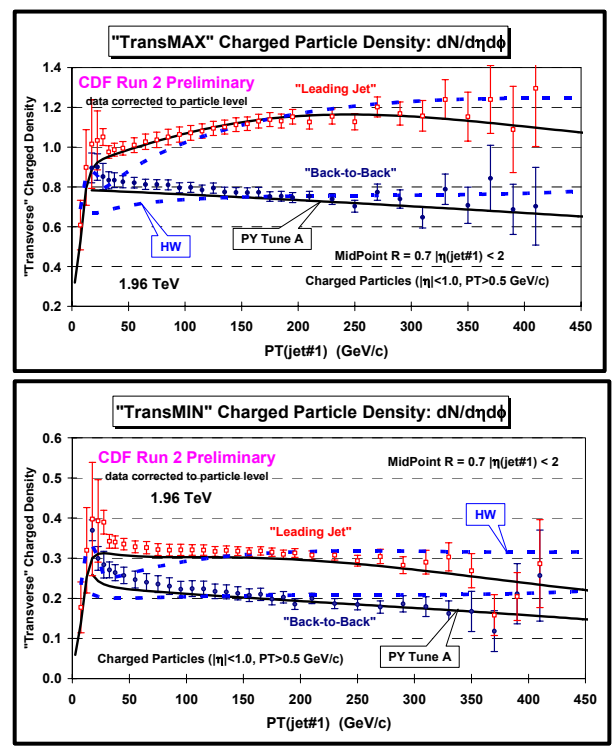

Fig. 1. CDF Run 2 data at $1.96 \mathrm{TeV}$ on charged particle density, $\mathrm{dN} / \mathrm{d} \eta \mathrm{d} \phi$ with $\mathrm{p}_{\mathrm{T}}>0.5 \mathrm{GeV} / \mathrm{c}$ and $|\eta|<1$ in the "transMAX" region (top) and the "transMIN" region (bottom) for "leading jet" and "back-to-back" events as a function of the leading jet $\mathrm{P}_{\mathrm{T}}$ compared with PYTHIA Tune A and HERWIG (without MPI). more sensitive to the underlying event, while "transMAX" minus the "transMIN" (i.e. "transDIF") is more sensitive to initial and final-state radiation. This idea, was first suggested by Bryan Webber, and implemented by in a paper by Jon Pumplin [1]. Also, Valaria Tano studied this in her CDF Run 1 analysis of maximum and minimum transverse cones [2].

Fig. 1 and Fig. 2 show the CDF Run 2 data on the density of charged particles and the

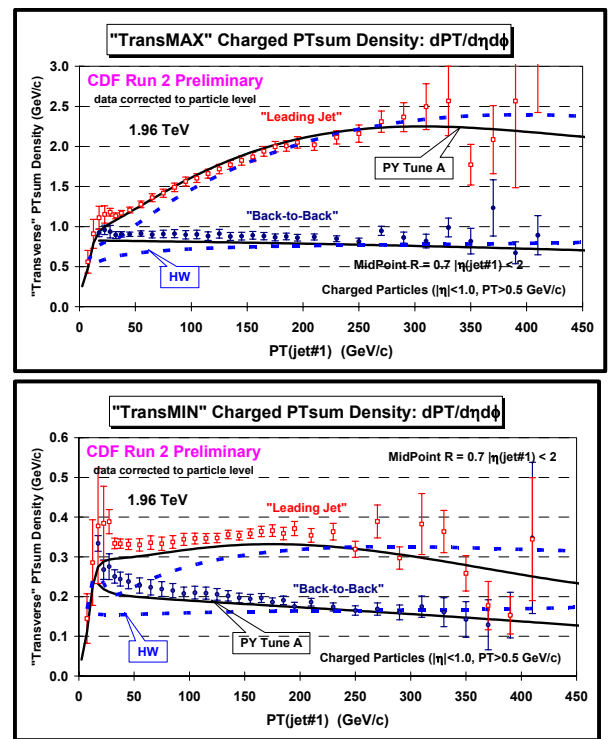

Fig. 2. CDF Run 2 data at $1.96 \mathrm{TeV}$ on scalar PTsum density of charged particles, $\mathrm{dPT} / \mathrm{d} \eta \mathrm{d} \phi$ with $\mathrm{p}_{\mathrm{T}}>0.5 \mathrm{GeV} / \mathrm{c}$ and $|\eta|<1$ in the "transMAX" region (top) and the

"transMIN" region (bottom) for "leading jet" and "back-toback" events as a function of the leading jet $\mathrm{P}_{\mathrm{T}}$ compared with PYTHIA Tune A and HERWIG (without MPI).

charged PTsum density in the "transMAX" and "transMIN" regions for "leading jet" and "back-to-back" events. The data are corrected to the particle level (with errors that include both the statistical error and the systematic uncertainty) and compared with PYTHIA Tune A (with MPI) and HERWIG [3] (without MPI) at the particle level (i.e. generator level). PYTHIA Tune A was determined by fitting the CDF Run 1 "underlying event" data [4].

As expected, the "leading jet" and "backto-back" events behave quite differently. For 
the "leading jet" case the "transMAX" densities rise with increasing $\mathrm{P}_{\mathrm{T}}(\mathrm{jet} \# 1)$, while for the "back-to-back" case they fall with increasing $\mathrm{P}_{\mathrm{T}}(\mathrm{jet} \# 1)$. The rise in the "leading jet" case is, of course, due to hard initial and final-state radiation, which has been suppressed in the "back-to-back" events. The "back-to-back" events allow for a closer look at the underlying event and PYTHIA Tune A does a better job describing the data than HERWIG.
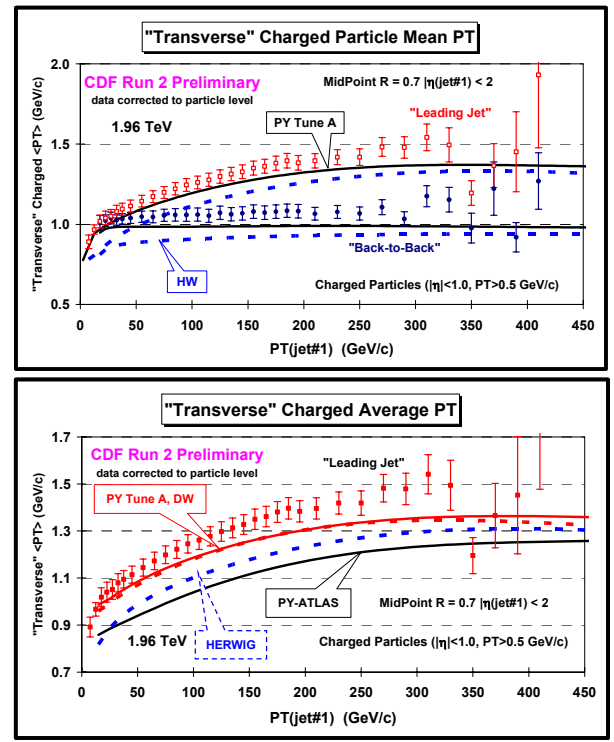

Fig. 3. (top) CDF Run 2 data at $1.96 \mathrm{TeV}$ average $\mathrm{p}_{\mathrm{T}}$ of charged particles with $\mathrm{p}_{\mathrm{T}}>0.5 \mathrm{GeV} / \mathrm{c}$ and $|\eta|<1$ in the overall "transverse" region for "leading jet" and "back-toback" events as a function of the leading jet $\mathrm{P}_{\mathrm{T}}$ compared with PYTHIA Tune A and HERWIG. (bottom) CDF Run 2 data at $1.96 \mathrm{TeV}$ on the average $\mathrm{pT}$ of charged particles with $\mathrm{p}_{\mathrm{T}}>0.5 \mathrm{GeV} / \mathrm{c}$ and $|\eta|<1$ in the overall "transverse" region for "leading jet" events compared with PYTHIA Tune A, Tune DW, HERWIG, and the ATLAS PYTHIA Tune.

The "transMIN" densities are more sensitive to the underlying event and the "back-to-back" data show a decrease in the "transMIN" densities with increasing $\mathrm{P}_{\mathrm{T}}(\mathrm{jet} \# 1)$, which is described fairly well by PYTHIA Tune A but not by HERWIG (without MPI). The decrease of the "transMIN" densities with increasing $\mathrm{P}_{\mathrm{T}}(\mathrm{jet} \# 1)$ for the "back-to-back" events is very interesting and might be due to a "saturation" of the multiple parton interactions at small impact parameter. Such an effect is included in PYTHIA Tune A but not in HERWIG (without MPI).

\begin{abstract}
Table 1. Parameters for several PYTHIA 6.2 tunes using CTEQ5L. Tune A is a CDF Run 1 "underlying event" tune. Tune DW and DWT are CDF Run 2 tunes which fit the existing Run 2 "underlying event" data and fit the Run 1 Z-boson $\mathrm{p}_{\mathrm{T}}$ distribution. The ATLAS Tune is the default tune currently used by ATLAS at the LHC.
\end{abstract}

\begin{tabular}{|l|c|c|c|c|}
\hline Parameter & $\begin{array}{c}\text { Tune } \\
\text { A }\end{array}$ & $\begin{array}{c}\text { Tune } \\
\text { DW }\end{array}$ & $\begin{array}{c}\text { Tune } \\
\text { DWT }\end{array}$ & ATLAS \\
\hline MSTP(81) & 1 & 1 & 1 & 1 \\
\hline MSTP(82) & 4 & 4 & 4 & 4 \\
\hline PARP(82) & 2.0 & 1.9 & 1.9409 & 1.8 \\
\hline PARP(83) & 0.5 & 0.5 & 0.5 & 0.5 \\
\hline PARP(84) & 0.4 & 0.4 & 0.4 & 0.5 \\
\hline PARP(85) & 0.9 & 1.0 & 1.0 & 0.33 \\
\hline PARP(86) & 0.95 & 1.0 & 1.0 & 0.66 \\
\hline PARP(89) & 1800 & 1800 & 1960 & 1000 \\
\hline PARP(90) & 0.25 & 0.25 & 0.16 & 0.16 \\
\hline PARP(62) & 1.0 & 1.25 & 1.25 & 1.0 \\
\hline PARP(64) & 1.0 & 0.2 & 0.2 & 1.0 \\
\hline PARP(67) & 4.0 & 2.5 & 2.5 & 1.0 \\
\hline MSTP(91) & 1 & 1 & 1 & 1 \\
\hline PARP(91) & 1.0 & 2.1 & 2.1 & 1.0 \\
\hline PARP(93) & 5.0 & 15.0 & 15.0 & 5.0 \\
\hline
\end{tabular}

The average $\mathrm{p}_{\mathrm{T}}$ for charged particles with in the overall "transverse" region for "leading jet" and "back-to-back" events are compared with PYTHIA Tune A and HERWIG in Fig. 3. Both PYTHIA Tune A and HERWIG lie below the data, but PYTHIA Tune A does a much better job that HERWIG. HERWIG (without MPI) predicts a "softer" $\mathrm{p}_{\mathrm{T}}$ distribution of charged particles than is seen in the data.

Table 1 shows the parameters for several PYTHIA 6.2 tunes [5]. PYTHIA Tune DW is very similar to Tune A except that it also fits the CDF Run 1 Z-boson $\mathrm{P}_{\mathrm{T}}$ distribution which Tune A does not fit [6]. Tune DW also has $\operatorname{PARP}(67)=2.5$, which is the preferred value determined by $\mathrm{D} \varnothing$ in fitting their dijet $\Delta \phi$ distribution [7]. PARP(67) sets the high $\mathrm{p}_{\mathrm{T}}$ scale for initial-state radiation in PYTHIA. It determines the maximal parton virtuality 
allowed in time-like showers. Tune DW and Tune DWT are identical at $1.96 \mathrm{TeV}$, but Tune DW and DWT extrapolate differently to the LHC. Tune DWT uses the ATLAS energy dependence, $\operatorname{PARP}(90)=0.16$, while Tune DW uses the Tune A value of $\operatorname{PARP}(90)=$ 0.25. The ATLAS Tune is the default tune currently used by ATLAS at the LHC. All the tunes use the CTEQ5L structure functions.

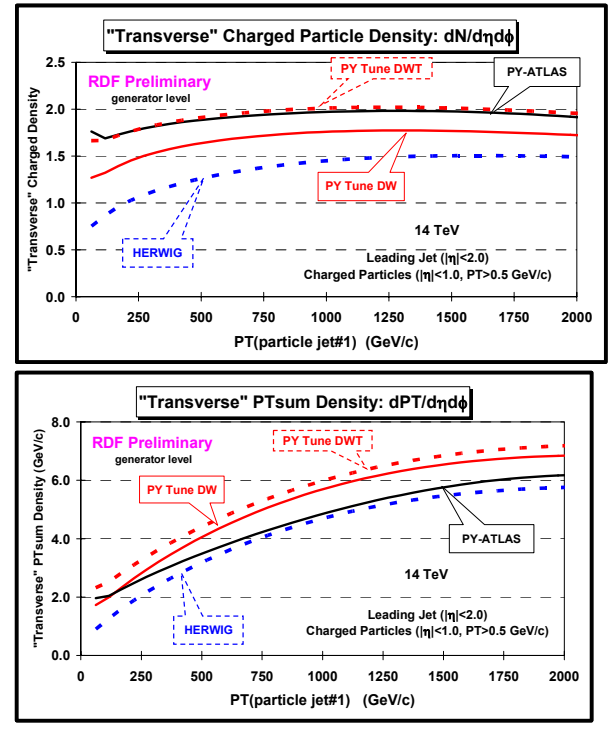

Fig. 4. Predictions at $14 \mathrm{TeV}$ of PYTHIA Tune DW, Tune DWT, HERWIG (without MPI), and the ATLAS Tune for the density of charged particles, $\mathrm{dN} / \mathrm{d} \eta \mathrm{d} \phi(t o p)$, and the charged PTsum density, dPT/ d $\eta \mathrm{d} \phi$ (bottom), with $\mathrm{p}_{\mathrm{T}}>0.5$ $\mathrm{GeV} / \mathrm{c}$ and $|\eta|<1$ in the overall "transverse" region for "leading jet" events as a function of the leading jet $\mathrm{P}_{\mathrm{T}}$.

PHYTIA Tune A agrees fairly well with the CDF Run 2 "underlying event" data for "leading jet" events and Tune DW roughly agrees with Tune A PYTHIA Tune A, Tune DW, and the ATLAS PYTHIA Tune predict about the same density of charged particles in the "transverse" region with $\mathrm{p}_{\mathrm{T}}>0.5 \mathrm{GeV} / \mathrm{c}$ for "leading jet" events at the Tevatron. However, the ATLAS Tune has a much softer $\mathrm{p}_{\mathrm{T}}$ distribution of charged particles resulting in a much smaller average $\mathrm{p}_{\mathrm{T}}$ per particles. Fig. 3 shows that the softer $\mathrm{p}_{\mathrm{T}}$ distribution of the ATLAS Tune does not agree with the CDF data.
Fig. 4 shows the predictions of PYTHIA Tune DW, Tune DWT, HERWIG (without MPI), and the ATLAS Tune for the density of charged particles and the PTsum density in the "transverse" region for "leading jet" events at the LHC. The PYTHIA Tunes (with MPI) predict a large increase in the charged particle density in going from the Tevatron to the LHC. HERWIG (without MPI) does not increase as much. At the LHC PYTHIA Tune DWT and the ATLAS Tune both predict about the same charged particle density with $\mathrm{p}_{\mathrm{T}}>0.5 \mathrm{GeV} / \mathrm{c}$, however, the ATLAS Tune predicts a smaller PTsum density than Tune DWT (i.e. the ATLAS Tune produces a softer $\mathrm{p}_{\mathrm{T}}$ distribution here as well).

In my opinion the best PYTHIA 6.2 tune at present is Tune DW or DWT. These tunes are identical at the $1.96 \mathrm{TeV}$ and they do a good job fitting the CDF Run 2 "underlying event" data. More work will have to be done in studying the "universality" of these tunes. For example, we do not know if Tune DW will correctly describe the underlying event in top quark production.

\section{References}

1. J. Pumplin, Phys. Rev. D57, 5787 (1998).

2. The CDF Collaboration, Phys. Rev. D70, 072002 (2004).

3. G. Marchesini and B. R. Webber, Nucl. Phys. B310, 461 (1988); I. G. Knowles, Nucl. Phys. B310, 571 (1988); S. Catani, G. Marchesini, and B. R. Webber, Nucl. Phys. B349, 635 (1991).

4. The CDF Collaboratio, Phys. Rev. D65, 092002 (2002).

5. T. Sjostrand, Phys. Lett. 157B, 321 (1985); M. Bengtsson, T. Sjostrand, and M. van Zijl, Z. Phys. C32, 67 (1986); T. Sjostrand and M. van Zijl, Phys. Rev. D36, 2019 (1987).

6. R. Field, to appear in the proceedings of the TeV4LHC workshop, Fermilab, 2006.

7. The DØ Collaboration, Phys. Rev. Lett. 94, 221801 (2005). 